\title{
Corrigendum to: Sporadic cases are the norm for complex disease
}

Jian Yang, Peter M Visscher and Naomi R Wray

European Journal of Human Genetics (2010) 18, 1044; doi:10.1038/ejhg.2009.233

Correction to: European Journal of Human Genetics (2010) 18, 1039-1043; doi:10.1038/ejhg.2009.177; published online 14 October 2009

Since the publication of the above paper, the authors noticed that there are some errors in the Appendix affecting the sense of this paper.

(1) In the lines just below the first equation in the Appendix (page 5), $x^{i}$ and $z^{i}$ should be $x_{i}$ and $z_{i}$.
(2) In the paragraph just below Equation (A1) in the Appendix (page 5):

- $\lambda_{n}=1-\Phi\left(T_{n}\right) / K$ should be $\lambda_{n}=\left[1-\Phi\left(T_{n}\right)\right] / K$

- $\lambda_{\mathrm{MZ}}=\lambda_{0}=\left(1-\Phi\left(T_{0}\right) / K\right)$ should be $\lambda_{\mathrm{MZ}}=\lambda_{0}=\left[1-\Phi\left(T_{0}\right)\right] / K$

- $H_{01}^{2}=\frac{\operatorname{cov}_{01}(\mathrm{MZ} \text { twins })}{K(1-K)}=\frac{\left[1-K-\Phi\left(T_{0}\right)\right]}{K(1-K)}$ should be $H_{01}^{2}=\frac{\operatorname{cov}_{01}(\mathrm{MZ} \text { twins })}{K(1-K)}=\frac{\left[1-K-\Phi\left(T_{0}\right)\right]}{(1-K)}$

The authors would like to apologise for this mistake. 